\title{
Effects of sex and APOE on Parkinson's Disease-related cognitive decline
}

\author{
Philip W. Tipton ${ }^{1}$, Nazli G. Bülbül ${ }^{2}$, Julia E. Crook ${ }^{3}$, Zachary Quicksall, Owen A. Ross ${ }^{2,4}$, \\ Ryan J. Uitti ${ }^{1}$, Zbigniew K. Wszolek ${ }^{1}$, Nilüfer Ertekin-Taner ${ }^{1,2}$ \\ ${ }^{1}$ Department of Neurology, Mayo Clinic, Mangurian Building, Jacksonville, Florida, USA \\ ${ }^{2}$ Department of Neuroscience, Mayo Clinic, Jacksonville, Florida, USA \\ ${ }^{3}$ Department of Health Sciences Research, Mayo Clinic, Jacksonville, Florida, USA \\ ${ }^{4}$ Department of Clinical Genomics, Mayo Clinic, Jacksonville, Florida, USA
}

\begin{abstract}
Introduction. Cognitive impairment is common in Parkinson's Disease, but the impact of predictive factors on incidence and rate of cognitive decline is incompletely understood.

We aimed to determine the effects of sex and APOE allele status on cognitive performance in patients with Parkinson's Disease (PD).

Material and methods. We conducted a retrospective analysis of 325 clinically diagnosed PD patients who underwent one or more cognitive screenings with a Mini-Mental Status Examination (MMSE) or Mattis Dementia Rating Scale (DRS-2). We used proportional odds regression models to estimate odds ratios for higher versus lower cognitive scores in association with age, sex, education, disease duration, and APOE allele status.

Results. Higher cognitive scores were independently associated with female sex on the MMSE (OR $2.43 ; 95 \% \mathrm{Cl} 1.14,5.14)$ and DRS-2 total (OR 4.14; 95\% Cl 2.01, 8.53). APOE $\varepsilon 4$ dose was associated with lower DRS-2 totals (OR 0.42; $95 \% \mathrm{Cl} 0.22,0.81)$, but there was no evidence of association with MMSE. Higher education level was also associated with higher scores on the MMSE (OR 1.22; $95 \% \mathrm{Cl} 1.07,1.38)$ and DRS-2 total (OR 1.31; $95 \% \mathrm{Cl} 1.15,1.50)$. Disease duration was not associated with cognitive performance on any measure when adjusting for age.

Conclusion. Male sex and APOE $\varepsilon 4$, along with age and lower education level, were associated with poorer cognitive performance among a population of predominantly non-demented PD patients.
\end{abstract}

Key words: Parkinson's Disease, cognition, sex, genetics, APOE

(Neurol Neurochir Pol 2021; 55 (6): 559-566)

\section{Introduction}

Parkinson's Disease (PD) is a neurodegenerative movement disorder with substantial phenotypic heterogeneity extending beyond motor symptoms. Cognitive impairment is common among those with PD and is associated with reduced quality of life (QoL) and increased caregiver stress/burden [1]. Mild cognitive impairment (MCI) is present in approximately $27 \%$ of non-demented individuals with PD and can occur within the first years of disease [2]. PD-MCI has been identified in 10-42\% of patients within the first years of disease [3-6]. While PD-MCI is associated with an annual dementia conversion rate of approximately 6-9\%, some individuals remain cognitively stable, and others even revert to normal cognition [6,7]. Numerous studies have aimed to characterise the PD cognitive profile; however, definitive conclusions remain largely elusive due to co-occurring pathology, varying study methods, and intrinsic disease heterogeneity $[5,8]$. The establishment of diagnostic criteria for PD-D and PD-MCI has significantly improved the quality of data, allowing for more cross-study comparisons $[9,10]$.

Address for correspondence: Nilüfer Ertekin-Taner, Department of Neurology, Mayo Clinic, 4500 San Pablo Rd., Jacksonville, FL 32224, USA;

e-mail: taner.nilufer@mayo.edu

Received: 26.05.2021 Accepted: 8.08.2021 Early publication date: 13.10.2021

This article is available in open access under Creative Common Attribution-Non-Commercial-No Derivatives 4.0 International (CC BY-NC-ND 4.0) license, allowing to download articles and share them with others as long as they credit the authors and the publisher, but without permission to change them in any way or use them commercially. 
Numerous studies have aimed to identify risk factors for the incidence and progression of PD-related cognitive decline. The frequency of PD-MCI appears to correlate with increasing age, disease duration, and disease severity [2]. PD$\mathrm{MCI}$, increasing age, male sex, and severity of PD motor signs increase the risk of PD-D [11] . The effect of these risk factors on continuous variables of cognitive decline and domain-specific decline are less well characterised. The impact of various genetic mutations on PD-related cognitive decline is equally complex, and definitive conclusions are often lacking. GBA mutations are associated with cognitive impairment in $\mathrm{PD}$; however [12], the role of other genetic factors is incompletely understood. APOE $\varepsilon 4$ allele increases the risk of dementia due to Alzheimer's Disease (AD); however, results are mixed when assessing the relationship to PD-related cognitive decline [12-15].

\section{Clinical rationale for study}

We aimed to determine the effect of sex and APOE allele status on cognitive performance within our PD cohort comprising 325 individuals who were predominantly dementia-free and who had had one or more cognitive assessment. Our findings reveal the impact of these variables, as well as of age and education, on cognitive function in PD patients. This work has implications for both future research studies and clinical assessment of cognition in PD.

\section{Materials and methods}

\section{Participants}

We previously described the motor features of a cohort consisting of 1,028 individuals with clinically diagnosed PD [16]. We reviewed the medical records of these individuals to identify those who underwent cognitive testing with at least one Mini-Mental Status Examination (MMSE) and/or a Mattis Dementia Rating Scale-2 (DRS-2). The majority of patients were followed routinely in our movement disorders clinic and underwent cognitive testing only when it was deemed clinically necessary by the movement disorders specialist. A small subset of these patients $(n=106)$ also had annual testing as part of a routine screening battery, regardless of their cognitive status.

The MMSE and DRS-2 tests were chosen because they were the most commonly administered cognitive screens in our entire clinical cohort. MMSE and DRS-2 total scores were extracted, as were DRS-2 domain scores (i.e. Attention, Initiation/Perseveration, Construction, Conceptualisation, and Memory). Demographic information, including sex, age, years of formal education and disease duration (defined as time from motor symptom onset) were extracted from the medical records, and APOE allele status was determined by Taqman ${ }^{\text {tw }}$ assays. This study was approved by our institutional review board, and all patients provided written informed consent for participation in this study.

\section{Statistics}

We used proportional odds models with patient-specific random effects to estimate odds ratios for high versus low cognition scores in association with some or all of age, sex, the interaction of age and sex, follow-up time and $A P O E$ genotype. The model was constructed using all available MMSE and DRS-2 scores. The proportional odds model is typically used for ordinal response variables and is similar to the more familiar logistic regression model with a binary response variable that categorises patients as having a high versus low cognitive score according to some fixed threshold. With the proportional odds models we do not need to specify a threshold; rather the model assumes that the odds ratios apply to any threshold. The odds ratio estimates from the model can thus be interpreted in the same way as those for a logistic regression model. A linear regression model was not appropriate primarily due to ceiling effects of the cognition scores with many persons achieving maximum or near-maximum scores (Fig. 1). More specifically, we let $Y_{i j}$ be the cognitive measure of patient $i$ at visit $j$, we let $a g e_{i j}$ and duration $_{i j}$, be the corresponding age and disease duration of the patient at the same visit, and we let be a binary indicator of female sex, the years of education and be the number of copies of the APOE $\varepsilon 4$ allele. We then modelled the log odds so that the score will be above versus below any threshold $c_{k}$, for example, as

$$
\begin{aligned}
& \log \left(\frac{P\left(Y_{i j} \geq c_{k}\right)}{P\left(Y_{i j}<c_{k}\right)}\right)=\alpha_{k}+\beta_{1} \text { sex }_{i}+\beta_{2} \text { age }_{i j}+\beta_{3} \text { female }_{i} \\
& \times \text { age }_{i j}+\beta_{4} \text { duration }_{i j}+\beta_{5} \text { educ }_{\mathrm{i}}+\beta_{6} \mathrm{APOE}_{i}+r_{i}
\end{aligned}
$$

where the $r_{i}$ terms are normally distributed random effects for each person. The random effects are included to account for correlations of the multiple cognition measures of each patient. Variations of this model were used for each analysis according to the cognitive outcome measure and covariates of interest. For example, the above form of the model was used to assess for evidence of an interaction of age and sex; to estimate the associations of cognition with age for males and females separately, the model was re-expressed by replacing the terms involving age with: $\beta_{2}{ }^{*}$ male $_{\mathrm{i}} \times$ age $_{\mathrm{ij}}$ $\times \beta_{3}{ }^{*}$ female $_{\mathrm{i}} \times$ age $_{\mathrm{ij}}$ where male $_{\mathrm{i}}=1-$ female $_{\mathrm{i}}$, so that $\beta_{2}{ }^{*}=\beta_{2}$ and $\beta_{3}{ }^{*}=\beta_{2}+\beta_{3}$ are the log odds ratios for females and males respectively.

\section{Results}

\section{Cohort demographics}

Our study cohort consisted of 233 males and 92 females with clinically diagnosed PD (Tab. 1), consistent with the higher male prevalence of this disease [17]. The mean age at study entry was c.70 years for both males and females. There was no significant difference in the percentage of individuals with $\geq 1$ APOE $\varepsilon 4$ allele in males vs. females. 


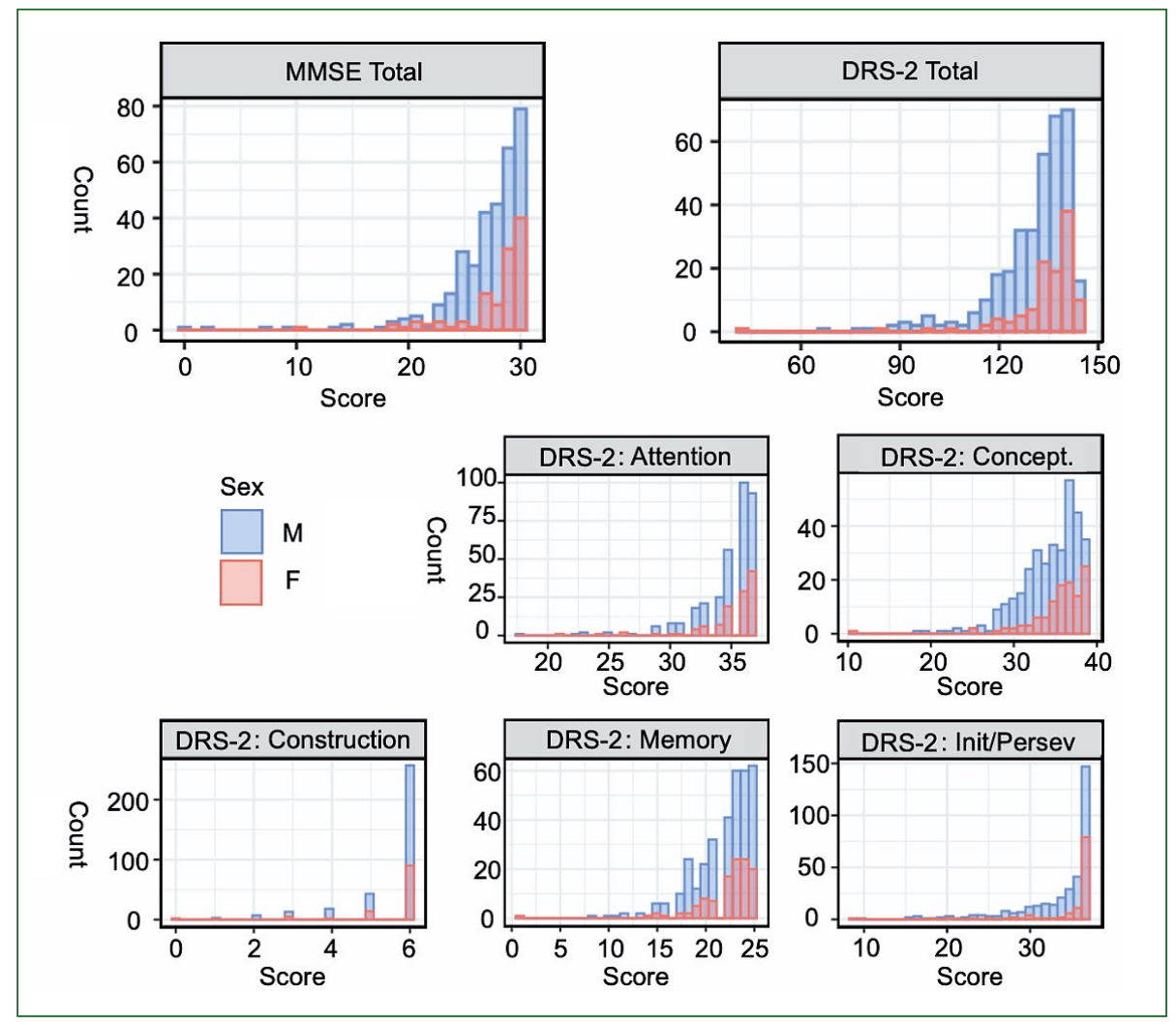

Figure 1. Histograms of all cognitive scores. Panels show score distribution for mini-mental status examination (MMSE), Mattis Dementia Rating Scale 2 (DRS-2) total, as well as each DRS-2 subscale stratified by sex

Table 1. Demographics of patients at first visit

\begin{tabular}{lccc} 
Variable & $\begin{array}{c}\text { Males } \\
(\mathbf{n = 2 3 3 )}\end{array}$ & $\begin{array}{c}\text { Females } \\
(\mathbf{n}=\mathbf{9 2})\end{array}$ & P-value \\
\hline Age, years & $70.4(9.9)$ & $69.4(9.4)$ & 0.394 \\
Formal education, years & $15.6(2.6)$ & $14.5(2.3)$ & 0.001 \\
APOE \&4 presence & $64(31.3)$ & $26(30.9)$ & 0.946 \\
$>1$ epoch testing & $133(56.8)$ & $41(44.6)$ & 0.047 \\
Duration of follow-up, days & $420(625)$ & $405(900)$ & 0.218 \\
Disease duration, years & $8.56(6.99)$ & $8.5(5.75)$ & 0.911 \\
MMSE: Total & $27.0(4.3)$ & $27.7(3.3)$ & 0.181 \\
DRS-2: Total & $128.8(12.9)$ & $132.1(14.5)$ & 0.105 \\
DRS-2: Attention & $34.7(3.1)$ & $35.0(3.2)$ & 0.536 \\
DRS-2: Conceptualisation & $34.0(3.8)$ & $35.0(4.2)$ & 0.080 \\
DRS-2: Construction & $5.5(1.1)$ & $5.5(1.1)$ & 0.751 \\
DRS-2: Initiation/Perseveration & $33.4(5.2)$ & $34.9(4.8)$ & 0.037 \\
DRS-2: Memory & $21.4(3.2)$ & $21.7(3.6)$ & 0.569
\end{tabular}

*Mean (standard deviation) or number (percent) are shown as appropriate

$A P O E \& 4$ allele heterozygotes and homozygotes totalled 84 and six, respectively. There were more males with $>1$ evaluation, reflecting not only the higher number of male participants but also the greater rate of follow-up in males
(57\%) vs. females (45\%). Among those patients with more than one evaluation, there was a wide range of follow-up times; however, the mean follow-up time was similar for both sexes. Mean disease duration was 8.5 years for both 
Table 2. Association of variables with cognitive test total scores

\begin{tabular}{|c|c|c|c|c|}
\hline \multirow[b]{2}{*}{ Variable } & \multicolumn{2}{|c|}{$\begin{array}{c}\text { DRS-2 Total } \\
\text { (260 patients, } 463 \text { visits) }\end{array}$} & \multicolumn{2}{|c|}{$\begin{array}{c}\text { MMSE Total } \\
\text { (217 patients, } 433 \text { visits) }\end{array}$} \\
\hline & OR (95\% Cl) & P-value & OR (95\% Cl) & P-value \\
\hline Female & $4.14(2.01,8.53)$ & $8.30 \mathrm{E}-05$ & $2.43(1.14,5.14)$ & 0.018 \\
\hline Age - 1 year (Females) & $0.91(0.85,0.96)$ & 0.0016 & $0.86(0.80,0.92)$ & $4.80 \mathrm{E}-06$ \\
\hline Age - 1 year (Males) & $0.87(0.83,0.91)$ & $2.90 \mathrm{E}-10$ & $0.93(0.89,0.96)$ & $3.00 \mathrm{E}-05$ \\
\hline Disease duration -1 year & $0.96(0.91,1.01)$ & 0.12 & - & - \\
\hline Educational level - 1 year & $1.31(1.15,1.50)$ & $3.80 \mathrm{E}-05$ & $1.22(1.07,1.38)$ & 0.0018 \\
\hline APOE \&4 (dose) & $0.42(0.22,0.81)$ & 0.0077 & $0.78(0.40,1.51)$ & 0.46 \\
\hline
\end{tabular}

A proportional odds model with patient-specific random effects was used to model the odds of a patient having a DRS- 2 score above versus below an arbitrary threshold as. The 's are log odds ratios; thus thei estimates and confidence intervals were exponentiated to obtain odds ratio estimates. The model for MMSE was the same, except that disease duration was omitted due to a lack of convergence when fitting the model with it included

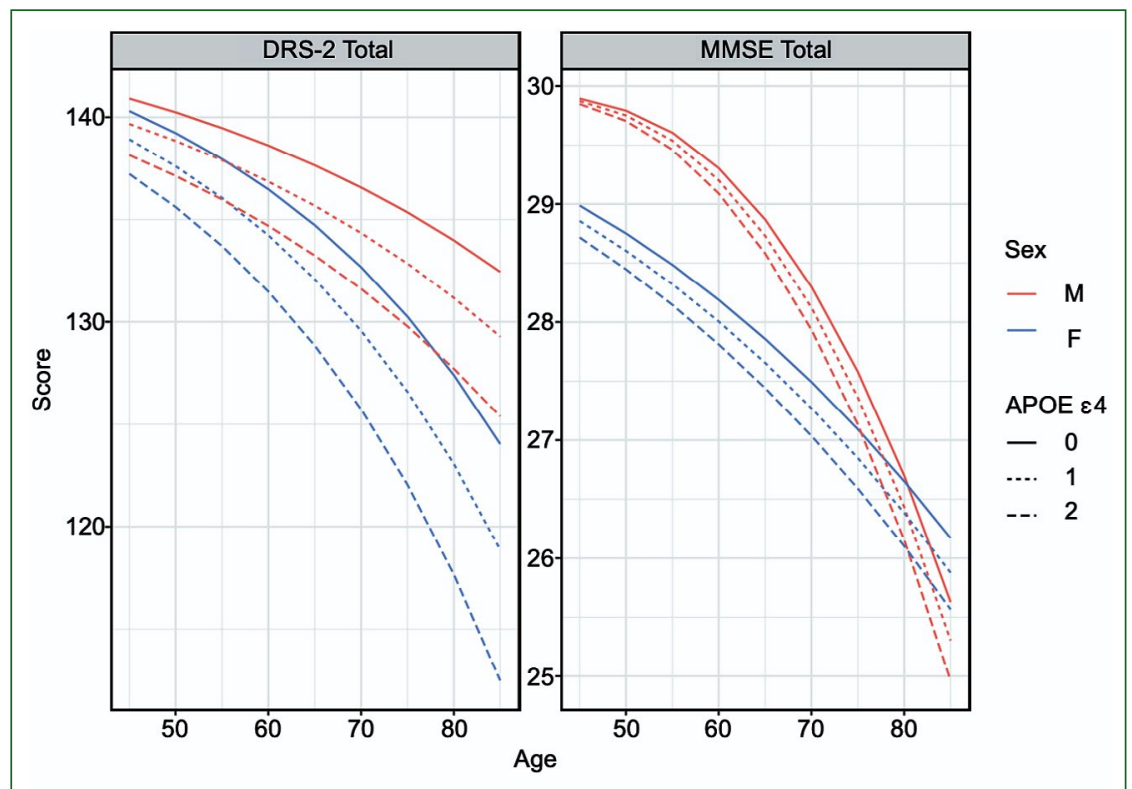

Figure 2. Trajectory of cognition with age by sex and APOE genotype. Estimated odds ratios from proportional odds models described in manuscript were used to obtain fitted trajectories of DRS-2 and MMSE with age, by sex and APOE $\varepsilon 4$ allele count

males and females. Males were more educated than females in this cohort, with 15.6 vs. 14.5 mean years of formal education, respectively.

\section{Cognitive scores}

Baseline cognitive scores obtained at first cognitive evaluation were similar for males and females (Tab. 1) with the exception of the DRS-2: Initiation/Perseveration sub-category where females scored higher than males. There was also a trend for higher mean baseline DRS-2: Conceptualisation scores in females, but this did not reach statistical significance. Applying the commonly accepted MMSE dementia threshold of 24, we found that $91 \%$ ( $n=199$ ) of participants were non-demented PD patients
(Fig. 1) [18]. This was mirrored by baseline DRS-2 total scores, in which $81 \%(\mathrm{n}=211)$ participants were above the dementia threshold of 123 [19].

We first assessed the association of sex, age, disease duration, education and APOE with DRS-2 and MMSE total scores (Tab. 2). Females had higher cognitive scores on both the MMSE (OR 2.43; 95\% CI 1.14, 5.14) and DRS-2 (OR 4.14; 95\% CI 2.01, 8.53) (Tab. 2). Age was associated with lower DRS-2 and MMSE scores in both males and females; estimated effect sizes were similar in both sexes for DRS-2, though there was evidence of an interaction of the effects of sex and age on MMSE ( $p=0.035$ ), with a lower odds ratio estimate for females, on the MMSE (OR 0.86; 95\% CI 0.80, 0.92 ), corresponding with faster decline (Tab. 2, Fig. 2). Education was associated with higher cognitive scores for both 
MMSE (OR 1.22; 95\% CI 1.07, 1.38) and DRS-2 total (OR 1.31; $95 \%$ CI 1.15, 1.50). APOE 44 dose was associated with lower scores on both tests, but reached statistical significance only with DRS-2 total scores (OR 0.42; 95\% CI 0.22, 0.81). There was no evidence of association between disease duration and cognitive performance.

We also assessed the DRS-2 sub-tests for their associations with the same variables (Supplemental Material). Females had higher cognitive scores on all sub-tests, reaching significance for Conceptualisation (OR 3.07, 95\% CI 1.64, 5.76) and Initiation/Perseveration (OR 7.60, 95\% CI 2.94, 19.65). Age was significantly associated with lower scores on all DRS-2 subtests both for males and females, with the exception of the DRS-2: Memory subscore in females. As with the total DRS-2 scores, after adjusting for age and the other variables, disease duration was not significantly associated with DRS-2 subscores, even though the effect estimates were suggestive of lower scores with higher disease duration. Education had a significant positive effect on all DRS- 2 subscores. APOE $\varepsilon 4$ dose had a significant lowering effect on DRS-2 Attention (OR 0.59; 95\% CI 0.37, 0.97 ) and DRS-2 Initiation/Perseveration (OR 0.44; 95\% CI $0.21,0.95$ ), along with lower but statistically insignificant effects on other subtests.

\section{Discussion}

Understanding the factors related to cognition in PD helps to inform clinicians to better counsel their patients and researchers to more reliably appreciate deviations from the typical disease phenotype. This also has implications for monitoring treatment response in clinical trials. We sought to contribute to this understanding by reporting cognitive screening results of 325 individuals clinically diagnosed with $\mathrm{PD}$, of whom more than half had longitudinal follow-up data. The majority of our cohort were non-demented and had mean disease duration of 8.5 years. Hence, our results provide information on relatively early cognitive changes which occur in PD.

We found females to have higher scores on MMSE, DRS-2 total and DRS-2 subscales, that were most pronounced for Conceptualisation and Initiation/Perseveration. These results could not be explained by disease duration, duration of follow-up or APOE \&4, which were not significantly different between the sexes. Further, males were more educated than females in this cohort. Baseline cognitive scores were also not different, with the exception of DRS-2: Initiation/Perseveration that was higher in females.

Collectively, these results suggest that male PD patients may be more susceptible to cognitive decline, especially in executive domains, and that these cognitive changes may be occurring relatively early in the disease course. Our results highlight the importance of monitoring and formally testing for PD-related cognitive decline, which may be more pronounced in males.
Our results differ from the conclusions of the Chinese Parkinson Study Group, which found that males scored higher on the MMSE, although males also had lower depression rates; education level was unreported [20]. Our cohort consisted of a roughly 2:1 male-to-female ratio, which is consistent with previous sex-related prevalence differences [12]. Our findings are consistent with other studies which showed sex-related cognitive associations in PD [11, 21, 22]. An Italian study of 6,599 idiopathic PD patients found male sex to be an independent risk factor for the development of Parkinson's Disease-Dementia (PD-D) [21]. This was validated by a recent meta-analysis of eight studies that showed independent associations with male sex [11]. A separate study of 84 PD patients found males to have greater impairments in executive function and processing speed, as well as a steeper rate of cognitive decline, compared to females [22]. This same study did not demonstrate a sex-related difference among controls. Future studies in longitudinally followed PD patients with no baseline dementia are needed to establish sex-related differences in cognitive decline, especially early in the disease process.

Despite being one of the most studied genes in $\mathrm{PD}$, the effect of $A P O E$ allele status on PD-related cognitive changes remains unclear. This is partly due to the heterogeneity of methodologies, specifically the use of different cognitive measures and inconsistently defined cognitive endpoints across studies. Several studies that have assessed cognition with the MMSE, albeit with different cutoff scores, have found no association with this cognitive measure and APOE genotypes [23-28]. In contrast, others have identified an association between $A P O E \& 4$ allele and the development of PD-D [29-30]. One study concluded that the MMSE was an inadequate screening measure for PD-MCI [31]. Studies using more detailed neuropsychological testing suggest that $A P O E \varepsilon 4$ is a risk factor for $\mathrm{PD}-\mathrm{D}$, and specifically for lower cognitive performances on measures of memory, attention, executive function and language processing [32, 33]. Most recently, a meta-analysis of 57 studies found that the presence of an APOE $\varepsilon 4$ allele was associated with PD-related cognitive impairment (Risk Ratio 3.04) [34]. More broadly, we have previously shown that the $A P O E \varepsilon 4$ allele is associated with lower baseline memory and increased decline in non-demented individuals not selected for the presence of $\mathrm{PD}$ [35]. It remains unclear whether an $A P O E$ $\varepsilon 4$ dose effect is greater in individuals with $P D$; however, recent work has shown that APOE $\varepsilon 4$ exacerbates a-synuclein [36].

Our findings in this study demonstrate the adverse effects of $A P O E \& 4$ on cognition in predominantly non-demented individuals with $\mathrm{PD}$ relatively early in their clinical course.

In our cohort, we determined that APOE $\varepsilon 4$ was associated with lower cognitive scores, which achieved statistical significance for DRS-2 total as well as subscale scores of attention and initiation/perseveration. Our results are consistent with another study which identified that the APOE $\varepsilon 4$ allele was associated with lower cognitive performance on the DRS-2 among PD patients [37]. More recently, a study showed steeper declines on 
the Montreal Cognitive Assessment in patients with clinically diagnosed early PD AOPE $\varepsilon 4$ allele carriers [14]. The DRS- 2 is one of the most commonly used global cognitive assessments for PD, and has been recommended by the Movement Disorders Society Task Force for the evaluation of PD-MCI [9], although the exact cognitive screening test most suitable for assessment in PD patients remains unclear. A recent study found that a DRS- 2 cutoff of 138 was estimated to be $87.5 \%$ sensitive and $68.9 \%$ specific for PD-related cognitive impairment, with single domain impairment sensitivity highest for executive dysfunction [38]. Trends of cognitive impairment and decline were similar on the MMSE and DRS-2 totals for our cohort, although score distributions reveal less of a ceiling effect with the DRS-2, suggestive of a greater utility in detecting cognitive decline especially early in the disease process.

We found that a higher education level was associated with higher MMSE, DRS-2 total and all DRS-2 subscale scores. There is some disagreement regarding the protective effect of education against PD-related cognitive impairment. One study found that education level did not predict the occurrence of PD-D during a five year follow up [39], whereas others have associated lower education levels with poorer cognitive performance [2]. A protective effect of higher education against all cause dementia is generally accepted [40].

This study has several limitations, most of which are intrinsic to its retrospective chart review design. Cognitive screening was not routinely performed on patients within our cohort as evidenced by only 325 patients with $\geq 1$ MMSE or DRS-2 among 1,028 total PD patients whose charts were reviewed. Therefore, our data is subject to sampling bias for those patients with a pre-test suspicion of cognitive impairment. Nevertheless, cognitive score distributions indicate that our cohort was largely non-demented based on traditional cutoff scores (i.e. MMSE $>24$ and/or DRS-2 total $>123$ ). This skewed distribution of cognitive scores towards the non-demented range may have limited our ability to detect some risk factor associations that occur later in the PD-related cognitive decline process. Only a subset of the patients had $>1$ cognitive testing, and the average follow up time was less than 1.5 years with an average disease duration of 8.5 years. One study found a nonlinear rate of PD-related cognitive decline with an early phase of $<1$ point/yr on the MMSE followed by a more precipitous rate ( $\sim 3$ points/yr) beginning after the thirteenth year of disease duration [41]. Our short follow up interval at a relatively early disease phase may have been another limiting factor to detect a statistically significant association of cognitive decline in PD with disease duration. Previous studies have indicated that patients with $\mathrm{PD}-\mathrm{MCI}$ may remain stable, revert to normal cognition, or progress to dementia. The latter occurs at a rate of 6-9\% annually [6, 7]. Influences on progression are likely to be multifactorial, including genetic and lifestyle factors in addition to those directly related to the underlying pathology. Inclusion of MMSE as a metric for cognitive decline in PD may be viewed as a limitation, given previous reports that this is not a sensitive measure for detecting PD-MCI [31]. We included MMSE in this study given its common usage in the clinical setting but also incorporated DRS-2, which has been recommended by the Movement Disorders Society Task Force for the evaluation of PD-MCI [9]. Moreover, our results were congruent between these two cognitive tests although the study was not powered to formally compare them. Lastly, our model did not include medication details relative to the timing of cognitive testing. It is conceivable that this may have had some impact.

\section{Clinical implications/future directions}

We conclude that age, male sex, APOE $\varepsilon 4$ and a lower education level are associated with poorer cognitive performance among a population of largely non-demented PD patients relatively early in their clinical course. Our findings have implications for screening in PD for cognitive impairment by formal neuropsychological testing, even in the absence of clinical complaints. Such screens will provide further information on the trajectory of cognitive changes in $\mathrm{PD}$ and both risk and protective factors, that may be critical for the counselling of patients. Male PD patients may be more susceptible than females to cognitive decline, especially in the executive domain, something which cannot be explained by sex-differences in disease duration, age or APOE. Importantly, this susceptibility occurred despite higher education in males in our cohort. These findings should prompt future studies to explore the role of environmental and genetic factors in driving sex differences in cognitive decline in PD.

Acknowledgements: We thank Audrey Strongosky for her role in coordinating various aspects of the clinical enrollment for this study as well as the patients and their families for their participation; without them, these studies would not have been possible. Mayo Clinic is an American Parkinson Disease Association (APDA) Mayo Clinic Information and Referral Centre, an APDA Centre for Advanced Research and Mayo Clinic Lewy Body Dementia Association (LBDA) Research Centre of Excellence. NET is funded in part by NIH/NIA U01 AG046139, RF1 AG051504, and R01 AG061796. OAR is supported in part by the National Institutes of Health (NIH; R01 NS78086; U54 NS100693; U54 NS110435), the US Department of Defense (W81XWH-17-1-0249), Mayo Clinic Centre for Individualised Medicine, the Little Family Foundation, the Mangurian Foundation for Lewy Body Research, and the Functional Genomics of LBD Programme at the Mayo Clinic.

Funding: This research did not receive any specific grant from funding agencies in the public, commercial, or not-for-profit sectors. Conflict of the interest: $Z W$ received support from the Mayo Clinic Centre for Regenerative Medicine, and gifts from the Sol Goldman Charitable Trust, and the Donald G. and Jodi P. Heeringa Family, the Haworth Family Professorship in 
Neurodegenerative Diseases Fund, and the Albertson Parkinson's Research Foundation. He serves as PI or Co-PI on Biogen, Inc. (228PD201), Biohaven Pharmaceuticals, Inc. (BHV4157-206 and BHV3241-301), and Neuraly, Inc. (NLY01-PD-1) grants. He serves as PI of the Mayo Clinic American Parkinson Disease Association (APDA) Information and Referral Centre. He is co-editor-in-chief of Neurologia i Neurochirurgia Polska (Polish Journal of Neurology and Neurosurgery).

(C2021 Mayo Foundation for Medical Education and Research

\section{References}

1. Corallo F, De Cola MC, Lo Buono V, et al. Observational study of quality of life of Parkinson's patients and their caregivers. Psychogeriatrics. 2017; 17(2): 97-102, doi: 10.1111/psyg.12196, indexed in Pubmed: 27338524.

2. Litvan I, Aarsland D, Adler CH, et al. MDS Task Force on mild cognitive impairment in Parkinson's disease: critical review of PD-MCI. Mov Disord. 2011; 26(10): 1814-1824, doi: 10.1002/mds.23823, indexed in Pubmed: 21661055.

3. Aarsland D, Brønnick K, Larsen JP, et al. Norwegian ParkWest Study Group. Cognitive impairment in incident, untreated Parkinson disease: the Norwegian ParkWest study. Neurology. 2009; 72(13): 1121-1126, doi: 10.1212/01.wnl.0000338632.00552.cb, indexed in Pubmed: 19020293.

4. Weintraub D, Simuni T, Caspell-Garcia C, et al. Parkinson's Progression Markers Initiative. Cognitive performance and neuropsychiatric symptoms in early, untreated Parkinson's disease. Mov Disord. 2015; 30(7): 919-927, doi: 10.1002/mds.26170, indexed in Pubmed: 25737166.

5. Yarnall AJ, Breen DP, Duncan GW, et al. ICICLE-PD Study Group. Characterizing mild cognitive impairment in incident Parkinson disease: the ICICLE-PD study. Neurology. 2014; 82(4): 308-316, doi: 10.1212/ WNL.0000000000000066, indexed in Pubmed: 24363137.

6. Broeders M, de Bie RMA, Velseboer DC, et al. Evolution of mild cognitive impairment in Parkinson disease. Neurology. 2013; 81(4): 346-352, doi: 10.1212/WNL.0b013e31829c5c86, indexed in Pubmed: 23794682.

7. Pedersen KF, Larsen JP, Tysnes OB, et al. Natural course of mild cognitive impairment in Parkinson disease: A 5-year population-based study. Neurology. 2017; 88(8): 767-774, doi: 10.1212/ WNL.0000000000003634, indexed in Pubmed: 28108638.

8. Kehagia A, Barker R, Robbins T. Neuropsychological and clinical heterogeneity of cognitive impairment and dementia in patients with Parkinson's disease. The Lancet Neurology. 2010; 9(12): 1200-1213, doi: 10.1016/s1474-4422(10)70212-x.

9. Litvan I, Goldman JG, Tröster Al, et al. Diagnostic criteria for mild cognitive impairment in Parkinson's disease: Movement Disorder Society Task Force guidelines. Mov Disord. 2012; 27(3): 349-356, doi: 10.1002/mds.24893, indexed in Pubmed: 22275317.

10. Emre M, Aarsland D, Brown R, et al. Clinical diagnostic criteria for dementia associated with Parkinson's disease. Mov Disord. 2007; 22(12): 1689-707; quiz 1837, doi: 10.1002/mds.21507, indexed in Pubmed: 17542011.

11. Hoogland J, Boel JA, de Bie RMA, et al. MDS Study Group "Validation of Mild Cognitive Impairment in Parkinson Disease". Risk of Parkinson's disease dementia related to level I MDS PD-MCI. Mov Disord. 2019;
34(3): 430-435, doi: 10.1002/mds.27617, indexed in Pubmed: 30653248.

12. Fagan ES, Pihlstrøm L. Genetic risk factors for cognitive decline in Parkinson's disease: a review of the literature. Eur J Neurol. 2017; 24(4): 561-e20, doi: 10.1111/ene.13258, indexed in Pubmed: 28220571.

13. Iwaki H, Blauwendraat C, Leonard HL, et al. International Parkinson's Disease Genomics Consortium. Genomewide association study of Parkinson's disease clinical biomarkers in 12 longitudinal patients' cohorts. Mov Disord. 2019; 34(12): 1839-1850, doi: 10.1002/ mds. 27845, indexed in Pubmed: 31505070.

14. Kim R, Park S, Yoo D, et al. Impact of the apolipoprotein $E \varepsilon 4$ allele on early Parkinson's disease progression. Parkinsonism Relat Disord. 2021; 83: 66-70, doi: 10.1016/j.parkreldis.2021.01.004, indexed in Pubmed: 33484977.

15. Pierzchlińska A, Białecka M, Kurzawski M, et al. The impact of Apolipoprotein E alleles on cognitive performance in patients with Parkinson's disease. Neurol Neurochir Pol. 2018; 52(4): 477-482, doi: 10.1016/j.pjnns.2018.04.003, indexed in Pubmed: 29776682.

16. Konno T, Deutschländer A, Heckman MG, et al. Comparison of clinical features among Parkinson's disease subtypes: A large retrospective study in a single center. J Neurol Sci. 2018; 386: 39-45, doi: 10.1016/j.jns.2018.01.013, indexed in Pubmed: 29406964.

17. Haaxma CA, Bloem BR, Borm GF, et al. Gender differences in Parkinson's disease. J Neurol Neurosurg Psychiatry. 2007; 78(8): 819-824, doi: 10.1136/jnnp.2006.103788, indexed in Pubmed: 17098842.

18. Tombaugh TN, McIntyre NJ. The mini-mental state examination: a comprehensive review. J Am Geriatr Soc. 1992; 40(9): 922-935, doi: 10.1111/j.1532-5415.1992.tb01992.x, indexed in Pubmed: 1512391.

19. Llebaria G, Pagonabarraga J, Kulisevsky J, et al. Cut-off score of the Mattis Dementia Rating Scale for screening dementia in Parkinson's disease. Mov Disord. 2008; 23(11): 1546-1550, doi: 10.1002/ mds.22173, indexed in Pubmed: 18546326.

20. Song Y, Gu Z, An J, et al. Chinese Parkinson Study Group. Gender differences on motor and non-motor symptoms of de novo patients with early Parkinson's disease. Neurol Sci. 2014; 35(12): 1991-1996, doi: 10.1007/s10072-014-1879-1, indexed in Pubmed: 25012756.

21. Cereda E, Cilia R, Klersy C, et al. Dementia in Parkinson's disease: Is male gender a risk factor? Parkinsonism Relat Disord. 2016; 26: 67 72, doi: 10.1016/j.parkreldis.2016.02.024, indexed in Pubmed: 26952697.

22. Reekes $\mathrm{TH}$, Higginson $\mathrm{Cl}$, Ledbetter $\mathrm{CR}$, et al. Sex specific cognitive differences in Parkinson disease. NPJ Parkinsons Dis. 2020; 6: 7, doi: 10.1038/s41531-020-0109-1, indexed in Pubmed: 32284961.

23. Blázquez L, Otaegui D, Sáenz A, et al. Apolipoprotein E epsilon4 allele in familial and sporadic Parkinson's disease. Neurosci Lett. 2006; 406(3): 235-239, doi: 10.1016/j.neulet.2006.07.037, indexed in Pubmed: 16904828.

24. Jasinska-Myga B, Opala G, Goetz CG, et al. Apolipoprotein E gene polymorphism, total plasma cholesterol level, and Parkinson disease dementia. Arch Neurol. 2007; 64(2): 261-265, doi: 10.1001/archneur.64.2.261, indexed in Pubmed: 17296843.

25. Kurz MW, Dekomien G, Nilsen OB, et al. APOE alleles in Parkinson disease and their relationship to cognitive decline: a population-based, longitudinal study. J Geriatr Psychiatry Neurol. 2009; 22(3): 166-170, doi: 10.1177/0891988709332945, indexed in Pubmed: 19321880. 
26. Williams-Gray $\mathrm{CH}$, Goris An, Saiki M, et al. Apolipoprotein E genotype as a risk factor for susceptibility to and dementia in Parkinson's disease. J Neurol. 2009; 256(3): 493-498, doi: 10.1007/s00415009-0119-8, indexed in Pubmed: 19308307.

27. Ryu HG, Kwon OhD. Apolipoprotein E epsilon 4 allele is not associated with age at onset or MMSE of Parkinson's disease in a Korean study. Parkinsonism Relat Disord. 2010; 16(9): 615-617, doi: 10.1016/j. parkreldis.2010.06.015, indexed in Pubmed: 20674461.

28. Federoff M, Jimenez-Rolando B, Nalls MA, et al. A large study reveals no association between APOE and Parkinson's disease. Neurobiol Dis. 2012; 46(2): 389-392, doi: 10.1016/j.nbd.2012.02.002, indexed in Pubmed: 22349451.

29. Pankratz N, Byder L, Halter C, et al. Presence of an APOE4 allele results in significantly earlier onset of Parkinson's disease and a higher risk with dementia. Mov Disord. 2006; 21(1): 45-49, doi: 10.1002/ mds.20663, indexed in Pubmed: 16116614.

30. Gomperts SN, Locascio JJ, Rentz D, et al. Amyloid is linked to cognitive decline in patients with Parkinson disease without dementia. Neurology. 2013; 80(1): 85-91, doi: 10.1212/ WNL.0b013e31827b1a07, indexed in Pubmed: 23243071.

31. Hoops S, Nazem S, Siderowf AD, et al. Validity of the MoCA and MMSE in the detection of $\mathrm{MCl}$ and dementia in Parkinson disease. Neurology. 2009; 73(21): 1738-1745, doi: 10.1212/ WNL.0b013e3181c34b47, indexed in Pubmed: 19933974.

32. Mata IF, Leverenz JB, Weintraub D, et al. APOE, MAPT, and SNCA genes and cognitive performance in Parkinson disease. JAMA Neurol. 2014; 71(11): 1405-1412, doi: 10.1001/jamaneurol.2014.1455, indexed in Pubmed: 25178429.

33. Tsuang D, Leverenz JB, Lopez OL, et al. APOE $\varepsilon 4$ increases risk for dementia in pure synucleinopathies. JAMA Neurol. 2013; 70(2): 223228, doi: 10.1001/jamaneurol.2013.600, indexed in Pubmed: 23407718.
34. Guo Yu, Liu FT, Hou XH, et al. Predictors of cognitive impairment in Parkinson's disease: a systematic review and meta-analysis of prospective cohort studies. J Neurol. 2021; 268(8): 2713-2722, doi: 10.1007/s00415-020-09757-9, indexed in Pubmed: 32162063.

35. Carrasquillo MM, Crook JE, Pedraza 0, et al. Late-onset Alzheimer's risk variants in memory decline, incident mild cognitive impairment, and Alzheimer's disease. Neurobiol Aging. 2015; 36(1): 60-67, doi: 10.1016/j.neurobiolaging.2014.07.042, indexed in Pubmed: 25189118.

36. Zhao $\mathrm{Na}$, Attrebi ON, Ren Y, et al. APOE4 exacerbates $\alpha$-synuclein pathology and related toxicity independent of amyloid. Sci Transl Med. 2020; 12(529), doi: 10.1126/scitransImed.aay1809, indexed in Pubmed: 32024798.

37. Morley JF, Xie SX, Hurtig HI, et al. Genetic influences on cognitive decline in Parkinson's disease. Mov Disord. 2012; 27(4): 512-518, doi: 10.1002/mds.24946, indexed in Pubmed: 22344634.

38. Hendershott TR, Zhu D, Llanes S, et al. Comparative sensitivity of the MoCA and Mattis Dementia Rating Scale-2 in Parkinson's disease. Mov Disord. 2019; 34(2): 285-291, doi: 10.1002/mds.27575, indexed in Pubmed: 30776152.

39. Domellöf ME, Ekman U, Forsgren L, et al. Cognitive function in the early phase of Parkinson's disease, a five-year follow-up. Acta Neurol Scand. 2015; 132(2): 79-88, doi: 10.1111/ane.12375, indexed in Pubmed: 25644230.

40. Livingston G, Sommerlad A, Orgeta V, et al. Dementia prevention, intervention, and care. The Lancet. 2017; 390(10113): 2673-2734, doi: 10.1016/s0140-6736(17)31363-6.

41. Aarsland D, Muniz G, Matthews F. Nonlinear decline of mini-mental state examination in Parkinson's disease. Mov Disord. 2011; 26(2): 334-337, doi: 10.1002/mds.23416, indexed in Pubmed: 20960482. 